The Astrophysical Journal, 643:1166-1179, 2006 June 1

(C) 2006. The American Astronomical Society. All rights reserved. Printed in U.S.A.

\title{
DISCOVERY OF AN EXTRAORDINARILY MASSIVE CLUSTER OF RED SUPERGIANTS
}

\author{
Donald F. Figer, ${ }^{1}$ John W. MacKenty, ${ }^{2}$ Massimo Robberto, ${ }^{2}$ Kester Smith, ${ }^{2}$ \\ Francisco Najarro, ${ }^{3}$ Rolf P. Kudritzki, ${ }^{4}$ and Artemio Herrero ${ }^{5}$ \\ Received 2005 November 16; accepted 2006 February 7
}

\begin{abstract}
We report the discovery of an extraordinarily massive young cluster of stars in the Galaxy, having an inferred total initial cluster mass comparable to the most massive young clusters in the Galaxy. Using IRMOS, 2MASS, and Spitzer observations, we conclude that there are 14 red supergiants in the cluster, compared with five, in what was previously thought to be the richest Galactic cluster of such stars. We infer spectral types from near-infrared spectra that reveal deep $\mathrm{CO}$ bandhead absorption that can only be fit by red supergiants. We identify a gap of $\Delta K_{s} \sim 4$ mag between the stars and the bulk of the other stars in the region that can only be fit by models if the brightest stars in the cluster are red supergiants. We estimate a distance of $5.8 \mathrm{kpc}$ to the cluster by associating an $\mathrm{OH}$ maser with the envelope of one of the stars. We also identify a "yellow" supergiant of G6 I type in the cluster. Assuming a Salpeter IMF, we infer an initial cluster mass of 20,000-40,000 $M_{\odot}$ for cluster ages of 7-12 Myr. Continuing with these assumptions, we find that $80 \%$ of the initial mass and $99 \%$ of the number of stars remain at the present time. We associate the cluster with an X-ray source (detected by $A S C A$ and Einstein), a recently discovered very high energy $\gamma$-ray source (detected by INTEGRAL and HESS), and several nonthermal radio sources, finding that these objects are likely related to recent supernovae in the cluster. In particular, we claim that the cluster has produced at least one recent supernova remnant with properties similar to the Crab Nebula.
\end{abstract}

Subject headings: infrared: stars — stars: evolution — supergiants

\section{INTRODUCTION}

Massive stars have short lives and terminate their nuclear energy generation in supernovae near the massive stellar clusters in which they were born. The relationship between initial stellar mass and post-supernova end state depends on the mass lost during the pre-supernova phases, i.e., main-sequence, blue supergiant (BSG), red supergiant (RSG), and Wolf-Rayet phases (Heger et al. 2003). The mass of a star just before collapse as a supernova is the critical discriminant that determines the density of the end state, i.e., complete disruption, neutron star, black hole, and total implosion to black hole with no supernova. The hydrogen content determines spectroscopic features of supernovae. Type II-P/L supernovae are thought to be produced by progenitors with hydrogen-rich envelopes and Type $\mathrm{Ib} / \mathrm{c}$ supernovae by those without hydrogen (Heger et al. 2003). The mass of the envelope may also influence the spectroscopic properties of the supernovae; therefore, the wind-generated mass-loss history and/or mass loss driven by common envelope evolution may be important in this regard. Furthermore, rotation rate and metallicity may be important variables, as they affect wind-driven mass loss (Hirschi et al. 2004).

Evolutionary and core-collapse models predict that red supergiants may be the immediate predecessors to Type II-P supernovae (Heger et al. 2003), and there is supporting observational evidence for this scenario (Smartt et al. 2004; van Dyk 2005),

\footnotetext{
${ }^{1}$ Chester F. Carlson Center for Imaging Science, Rochester Institute of Technology, 54 Lomb Memorial Drive, Rochester, NY 14623-5604.

2 Space Telescope Science Institute, 3700 San Martin Drive, Baltimore, MD 21218; figer@stsci.edu.

3 Instituto de Estructura de la Materia, Consejo Superior de Investigaciones Cientificas, Calle Serrano 121, 28006 Madrid, Spain.

4 Institute for Astronomy, University of Hawaii, 2680 Woodlawn Drive, Honolulu, HI 96822.

5 Instituto de Astrofísica de Canarias, Via Láctea S/N, E-38200 La Laguna, Tenerife, Spain; and Departamento de Astrofísica, Universidad de La Laguna, Avenida Astrofísico Francisco Sánchez 2, E-38071 La Laguna, Tenerife, Spain.
}

although in one particularly famous example (1987A) the type is B3 I (Walborn et al. 1989). The observational evidence is necessarily quite limited, as red supergiants are relatively rare in the Galaxy. Indeed, there are only $\sim 200$ known in the Galaxy (Humphreys 1978; Garmany \& Stencel 1992; Levesque et al. 2005) and no more than five in any single coeval cluster (Beauchamp et al. 1994; Caron et al. 2003). This scarcity is understandable as a consequence of the Galactic cluster mass function, the stellar initial mass function (IMF), the presence of interstellar dust in the Galactic disk, and the shortness of the RSG phase predicted by stellar evolution models. In particular, the Galactic cluster mass function (Kharchenko et al. 2005) and stellar IMF (Salpeter 1955; Kroupa 2002) are both decreasing with mass, and RSGs only evolve from relatively massive stars having $M_{\text {initial }} \sim 8-25 M_{\odot}$ and ages of 6-15 Myr. Clearly, it is important to identify more RSGs, especially in coeval clusters, to test stellar evolution, core-collapse, and end-state predictions.

Much of our knowledge of RSG populations comes from studies of nearby galaxies and nearby regions in the Galaxy. McGregor \& Hyland (1984) and Massey \& Olsen (2003) discussed RSGs in open clusters in the Small Magellanic Cloud (SMC), the Large Magellanic Cloud (LMC), and the Galaxy. Humphreys (1970) listed RSGs in Galactic associations. Humphreys \& McElroy (1984) discussed the BSG to RSG ratio in Galactic clusters. Humphreys (1979a) gave histograms of the number of RSGs versus spectral type in the SMC, the LMC, and the Galaxy, showing that RSGs in the former are significantly earlier than in the latter two. Humphreys (1979b) gave a list of RSGs in the LMC. The highest number of such stars in any association in the LMC is eight. In the Galaxy, the Per OB1 association contains $\sim 20$ RSGs, but it is still unclear as to whether they form a group with a common, and coeval, formation history (Slesnick et al. 2002). The richest known coeval cluster of RSGs in the Galaxy is NGC 7419, with five (Caron et al. 2003).

It is interesting to analyze RSGs in coeval clusters because one can then be sure that observed differences between the stars 
TABLE 1

Supergiant Cluster Stars

\begin{tabular}{|c|c|c|c|c|c|c|c|c|}
\hline ID & 2MASS Name & $J$ & $H$ & $K_{s}$ & Spectral Type & $\begin{array}{c}\text { Obs. Date } \\
(2005)\end{array}$ & $M_{K_{s}}$ & $\log \left(L / L_{\odot}\right)$ \\
\hline $1 \ldots \ldots \ldots \ldots$ & $18375629-0652322$ & 9.748 & 6.587 & 4.962 & M3 I & Sep 16 & -11.60 & 5.6 \\
\hline ....... & $18375528-0652484$ & 9.904 & 6.695 & 5.029 & M4 I & Sep 16 & -11.53 & 5.6 \\
\hline  & $18375973-0653494$ & 9.954 & 6.921 & 5.333 & M4 I & Sep 16 & -11.23 & 5.5 \\
\hline 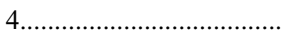 & $18375090-0653382$ & 9.658 & 6.803 & 5.342 & M0 I & Sep 16 & -11.22 & 5.5 \\
\hline  & $18375550-0652122$ & 10.547 & 7.178 & 5.535 & M6 I & Oct 13 & -11.03 & 5.4 \\
\hline 6. & $18375745-0653253$ & 9.866 & 7.038 & 5.613 & M5 I & Oct 13 & -10.95 & 5.4 \\
\hline 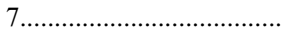 & $18375430-0652347$ & 9.941 & 7.065 & 5.631 & M2 I & Sep 16 & -10.93 & 5.4 \\
\hline$\ldots \ldots \ldots \ldots \ldots \ldots \ldots$ & $18375519-0652107$ & 10.772 & 7.330 & 5.654 & M3 I & Oct 13 & -10.91 & 5.4 \\
\hline 9 & $18375777-0652222$ & 10.262 & 7.240 & 5.670 & M3 I & Sep 16 & -10.89 & 5.3 \\
\hline 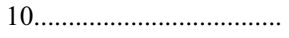 & $18375952-0653319$ & 10.179 & 7.218 & 5.709 & M5 I & Oct 13 & -10.85 & 5.3 \\
\hline 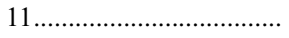 & $18375172-0651499$ & 10.467 & 7.325 & 5.722 & M1 I & Sep 16 & -10.84 & 5.3 \\
\hline 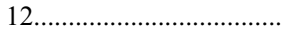 & $18380330-0652451$ & 10.143 & 7.238 & 5.864 & M0 I & Sep 16 & -10.70 & 5.3 \\
\hline 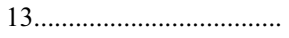 & $18375890-0652321$ & 10.907 & 7.716 & 5.957 & M3 I & Sep 15 & -10.61 & 5.2 \\
\hline  & $18374764-0653023$ & 10.495 & 7.576 & 6.167 & M1 I & Sep 16 & -10.40 & 5.4 \\
\hline 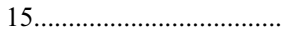 & $18375778-0652320$ & 10.651 & 8.070 & 6.682 & G6 I & Sep 15 & -9.88 & 5.0 \\
\hline
\end{tabular}

NotE.-The spectral types, absolute magnitudes, and luminosities are estimated in this paper.

are not simply related to age or metallicity effects. Of course, it is necessary to use massive clusters for such purposes, given that only the most massive clusters can have a significant number of RSGs. As an example, a cluster must have an initial mass at least as great as $10^{4} M_{\odot}$ to have more than $10 \mathrm{RSGs}$; it must also be at an age when its members will be in the RSG stage. In the Galaxy, there are only a few clusters with masses greater than $10^{4} M_{\odot}$. They are the Arches cluster (Figer et al. 2002), the Quintuplet cluster (Figer et al. 1999a, 1999b), the Central cluster (Figer 2004), and Westerlund 1 (Clark et al. 2005). In the former three cases, the clusters were identified by infrared observations for their close proximity to the Galactic center. In the latter case, the cluster was identified at optical wavelengths. This leaves a large portion of the Galaxy that is obscured at optical wavelengths but has yet to be probed.

Bica et al. (2003a, 2003b) and Dutra et al. (2003a) created a catalog of massive cluster candidates using an algorithm to search the Two Micron All Sky Survey (2MASS; Skrutskie et al. 1997, 2006). ${ }^{6}$ Most of these candidates are obscured at optical wavelengths, and some have already been shown to be massive stellar clusters (Dutra et al. 2003b; Bica et al. 2004; Borissova et al. 2005; Ivanov et al. 2005; Leistra et al. 2005).

Using this catalog, we have identified one of the most massive stellar clusters in the Galaxy. In this paper, we present infrared photometry and spectra, and an analysis using multiwavelength data sets that strongly suggests that there is a very massive young stellar cluster containing 14 RSGs near G25.25-0.15.

\section{OBSERVATIONS AND DATA REDUCTION}

Spectra were obtained in 2005 September and October of at the Kitt Peak National Observatory (KPNO) 2.1 and $4 \mathrm{~m}$ telescopes using the Infrared Multi-Object Spectrograph (IRMOS; MacKenty et al. 2004). The spectral resolution was $\sim 1000$, using a 3 pixel wide slit aperture. Calibration files (source darks, flat and Neon lamps, and relative darks) were taken immediately after the sequence of source exposures for the same slit configuration. The spectra were extracted from background-subtracted

6 This publication makes use of data products from 2MASS, which is a joint project of the University of Massachusetts and the Infrared Processing and Analysis Center/California Institute of Technology, funded by the National Aeronautics and Space Administration and the National Science Foundation. and flat-fielded images. The wavelength scale was fixed by relating pixel number to the locations of sky $\mathrm{OH}$ and neon emission lines in the calibration frames. The resulting signal-to-noise ratio was generally greater than 100 , as estimated from the data.

We also extracted 2MASS images and point-source photometry from 2MASS catalog, on a field centered at R.A. = $18^{\mathrm{h}} 37^{\mathrm{m}} 58^{\mathrm{s}}$, decl. $=-6^{\circ} 52^{\prime} 53^{\prime \prime}$. $(\mathrm{J} 2000.0)$. We obtained Spitzer Infrared Array Camera (IRAC) photometry through the Galactic Legacy Infrared Mid-Plane Survey Extraordinaire (GLIMPSE) point-source catalog (Benjamin et al. 2003). ${ }^{7}$

Table 1 lists the coordinates, magnitudes, spectral types, absolute infrared magnitudes, and luminosities for the target stars.

7 This work is based in part on observations made with the Spitzer Space Telescope, which is operated by the Jet Propulsion Laboratory, California Institute of Technology, under a contract with NASA.

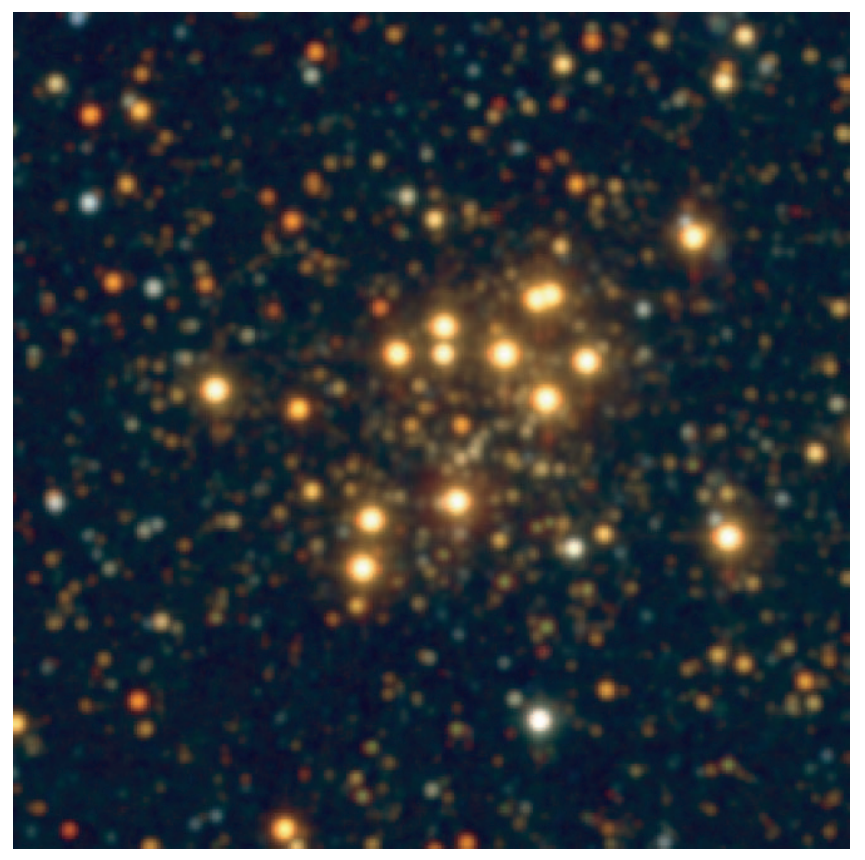

FIG. 1.-2MASS color composite image. 


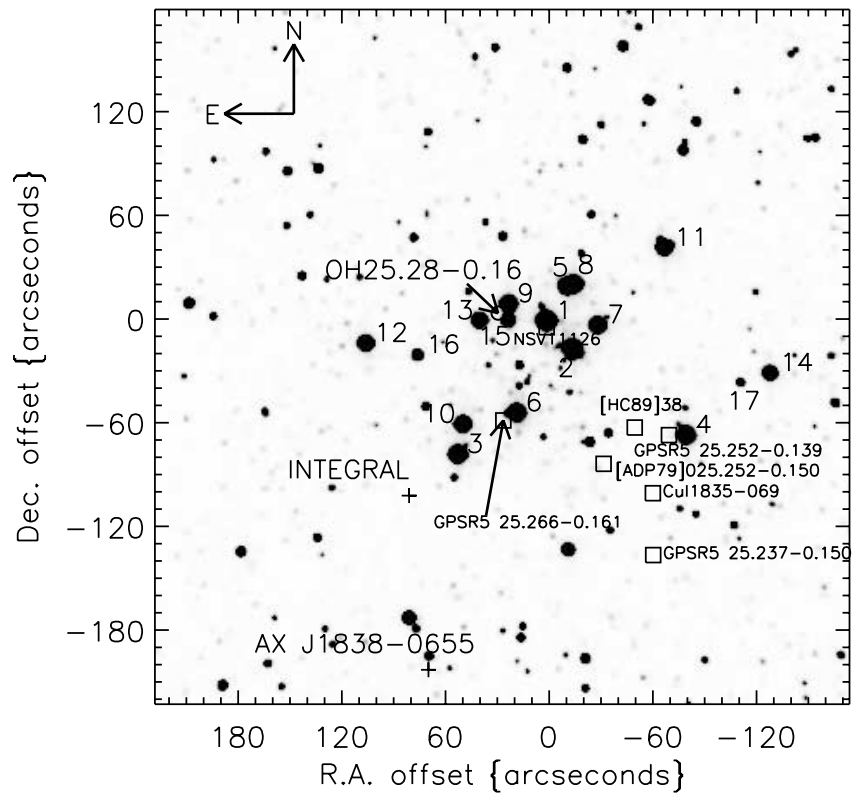

FIG. 2.-2MASS $K_{s}$-band image of cluster with offset coordinates from the brightest star in the cluster (No. 1). Stars are identified with numbers according to their IDs in Table 1. The maser $\mathrm{OH} 25.28-0.16$ is designated by an open circle, and its location has a positional uncertainty of $4^{\prime \prime}$ (Blommaert et al. 1994). The plus signs indicate the most likely positions of the INTEGRAL source and an $A S C A$ source, AX J1838.0-0655, with positional uncertainties of $3^{\prime}$ and $1^{\prime}$, respectively. Other objects, taken from SIMBAD, are designated by squares. Most of these objects are radio sources.

The coordinates are taken from the 2MASS Point Source Catalog, and the other quantities are determined in the remainder of this paper.

Figure 1 shows a color composite image from the 2MASS survey. Figure 2 shows a $K_{s}$-band image from the 2MASS survey, with designations for the objects in the table and objects extracted

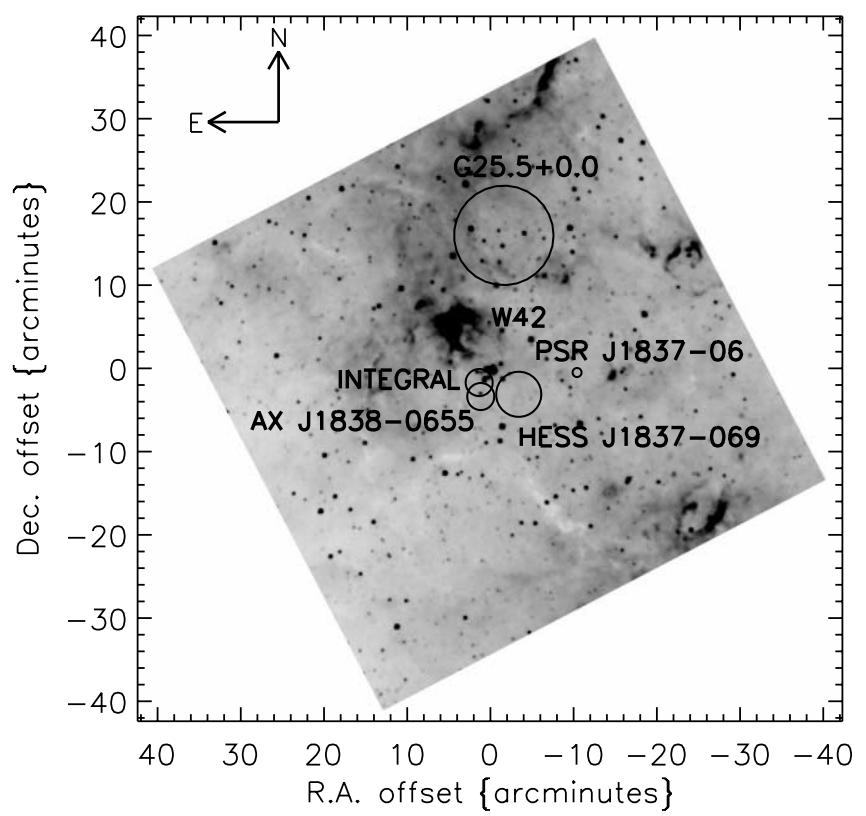

FIG. 3.- $M S X 8.3 \mu \mathrm{m}$ image of cluster with offset coordinates from the brightest star in the cluster (No. 1). The W42 star formation region is located about $5^{\prime}$ to the northeast of the cluster. The HESS source has a positional uncertainty of $1^{\prime}-2^{\prime}$ and a size of $7^{\prime}$. from SIMBAD. ${ }^{8}$ Many of these sources from the SIMBAD database were observed at X-ray, $\gamma$-ray, and radio wavelengths, as is discussed later in this paper. Figure 3 shows the region around the cluster at mid-infrared wavelengths, as observed by the Midcourse Space Experiment (MSX) mission (Price et al. 2001).

\section{ANALYSIS}

In this section, we estimate the spectral types, extinction, distance, and luminosities of the cluster stars.

\subsection{Spectral Types}

The brightest cluster stars appear to be RSGs, having an average spectral type of M3 I, consistent with the average type in the Galaxy of M2 I (Elias et al. 1985). Their spectra all have deep CO absorption near $2.2935 \mu \mathrm{m}$, and longer wavelengths, except for star 15, as shown in Figure 4. They are also much brighter than other stars in the region/cluster, as seen in Figures 2, 5, and 6 , and in Table 1 . We measure equivalent widths near the ${ }^{12} \mathrm{CO}$ band head between 2.290 and $2.320 \mu \mathrm{m}$, with the continuum level estimated as the average flux between 2.285 and $2.290 \mu \mathrm{m}$.

We can compare these measurements with those of template spectra from Kleinmann \& Hall (1986). As can be seen in Figure 7, the band head becomes stronger for later spectral types, and it is generally stronger for supergiants than for giants of a given subtype. These relationships are quantified in Figure 8. Considering our measurements, and the trends evident for the template stars, we conclude that the 14 brightest members of this cluster are all RSGs with spectral types of M0 I-M4 I. This is further supported by the fact that the latest spectral types appear to be redder, as expected (see Fig. 6). Overall, we estimate an error in the spectral types of a few subtypes.

${ }^{8}$ This research has made use of the SIMBAD database, operated at Centre de Données Astronomiques de Strasbourg, France.



FIG. 4.-IRMOS spectra of RSG cluster stars. The equivalent width of the ${ }^{12} \mathrm{CO}$ band head, in angstroms, and the corresponding spectral subtype are given. Note that all spectra show deep CO absorption, except for object 15, which is the next brightest star after the 14 brightest stars in the cluster. From its spectrum and photometric color we suggest that this star is a G-type supergiant. 


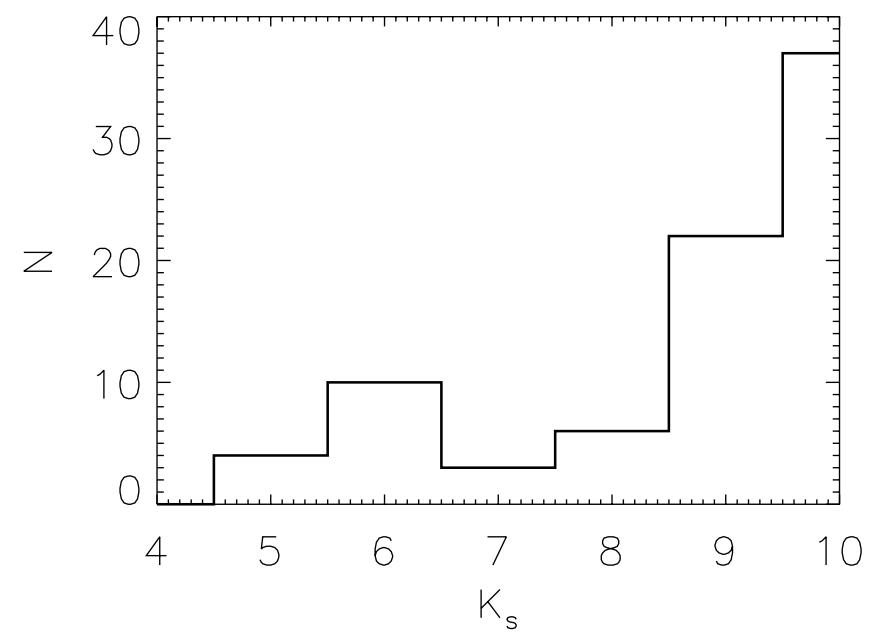

FIG. 5.-2MASS luminosity function of stars within $3^{\prime}$ of the center of the RSG cluster. The 14 RSGs are in the two brightest bins. Note the relatively large gap in brightness between the RSGs and the bulk of the fainter stars in the field. The sample has been culled of stars with reported errors greater than $0.1 \mathrm{mag}$ in $K_{s}$.
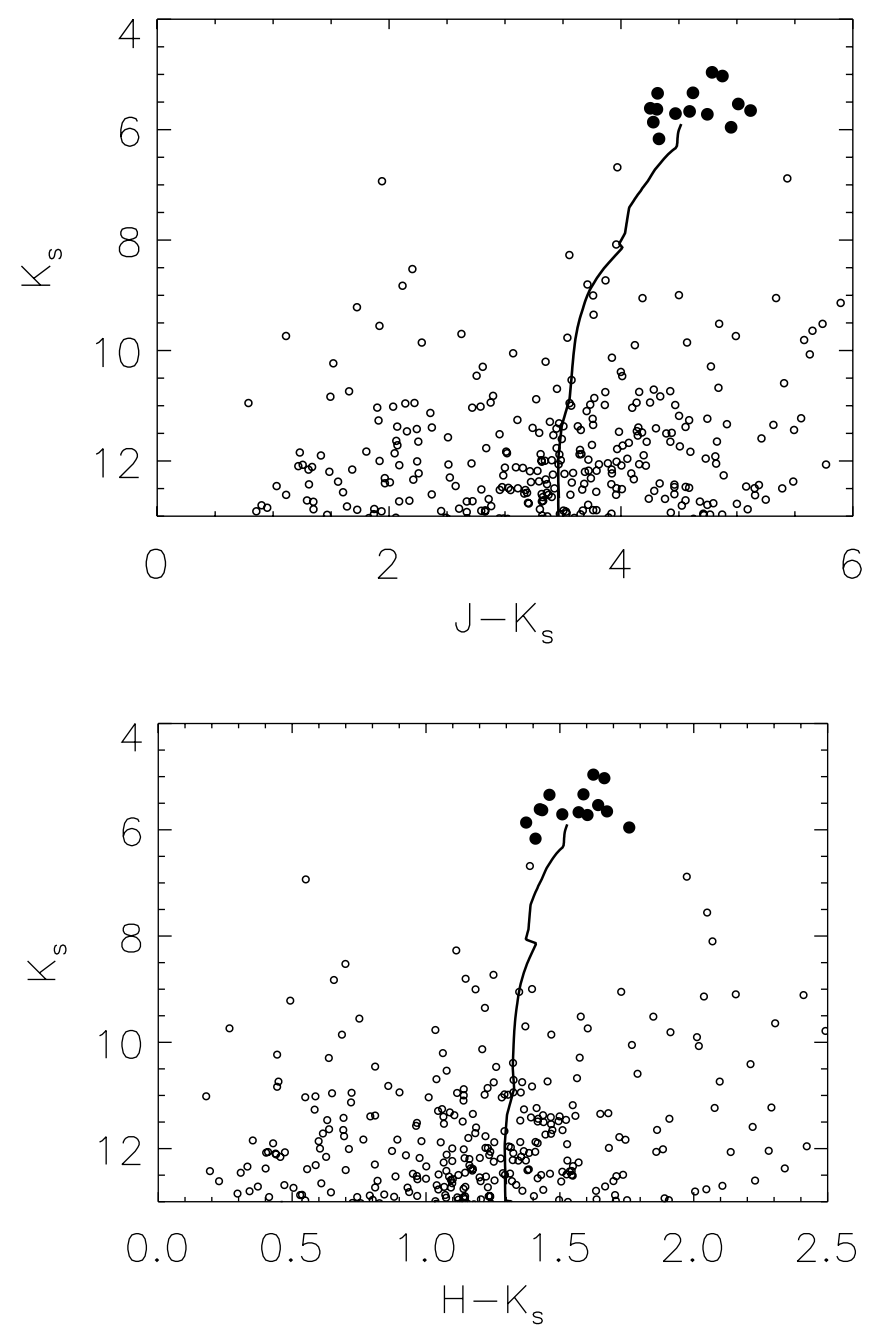

Fig. 6.-2MASS CMDs of stars within $3^{\prime}$ of the center of the RSG cluster showing the RSGs ( filled circles) and the fainter field stars (open circles). A $10 \mathrm{Myr}$ isochrone is overplotted, assuming the Geneva models with solar metallicity (Schaerer et al. 1993), a distance of $5.8 \mathrm{kpc}, A_{K_{s}}=2.74$, and the reddening law of Rieke et al. (1989). The color spread for the RSGs scales roughly with inferred spectral type; i.e., later types are redder. The sample has been culled of stars with reported errors greater than $0.1 \mathrm{mag}$ in $K_{s}$.
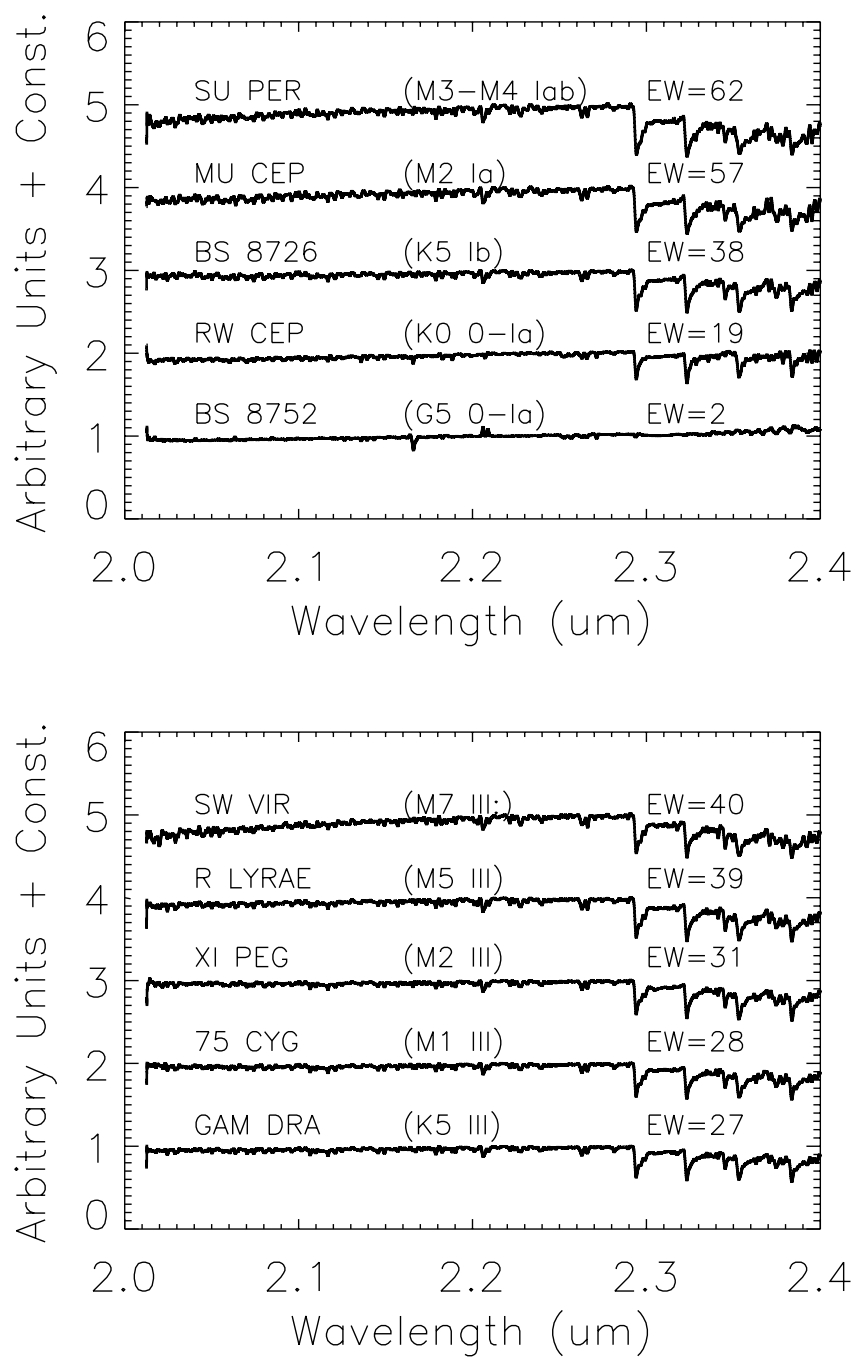

FIG. 7.- Spectra of template RSGs (top) and red giants (bottom) from Kleinmann \& Hall (1986). The equivalent width of the ${ }^{12} \mathrm{CO}$ band head, in angstroms, is given.



G5 G6 G7 G8 G9 K0 K1 K2 K3 K4 K5 MO M1 M2 M3 M4 M5 M6 Spectral Type

FIG. 8.-Relation between ${ }^{12} \mathrm{CO}$ equivalent width, as defined in the text, and spectral subtype for cluster stars (open circles), template RSGs (filled circles), and template red giants (squares). Lines are drawn through the best fits between equivalent width and subtype for the template stars. 



FIG. 9.-2MASS color-color diagrams of stars within $3^{\prime}$ of the center of the RSG cluster showing the RSGs ( filled circles) and the fainter field stars (open circles). A reddening vector is plotted from the expected intrinsic colors of RSGs, assuming the extinction law of Rieke et al. (1989). The sample has been culled of stars with reported errors greater than $0.1 \mathrm{mag}$ in $K_{\mathrm{s}}$. The bulk of the field stars are not as red as the RSGs and are thus likely in the foreground.

The spectrum for star 15 has weak (but nonzero) CO absorption, and this star is also fainter than the 14 brightest stars in the cluster. Its spectrum is more indicative of a mid G-type supergiant. We suggest that it is a G6 I type based on the weak CO features. Given the location of the star on the theoretical isochrones (see our Fig. 6; Schaerer et al. 1993), we suggest that it is a cluster member in transition to, or from, the RSG phase. While such stars are rare, we expect there to be of order one such star in a cluster with the mass and age we estimate (see below).

In any case, the stars fainter than $K_{s}=6.0$ in the region are not $\mathrm{M}$ supergiants. In Figure 6, one can identify a cluster of fainter stars in the color-magnitude diagram (CMD) starting at $K_{s} \sim 10$. These stars likely represent the main sequence. The expected brightness difference between the main-sequence stars and the RSGs is roughly equal to the observed difference. The faint cluster stars can also be seen in Figure 9, having colors between $H-K_{s}=1.1$ and 1.3; the RSGs are redder in both colors. The expected color difference between the main-sequence stars and the RSG stars is $H-K_{s} \sim 0.3$, in accordance with the measured difference. All the supergiant cluster stars lie on the reddening vector.

\subsection{Extinction}

We estimate extinction by adopting average intrinsic colors for M supergiants of $H-K=0.21$ and $J-K=0.89$ (Elias et al. 1985 ) and comparing these to the average colors of the RSGs in the cluster. We identify the difference between the two as the color excess and convert it into $A_{K_{s}}$ using the relation in Rieke et al. (1989), finding from $E_{H-K}$

$$
\begin{aligned}
A_{K_{s}} & =\frac{E_{H-K}}{\left[\left(\lambda_{H} / \lambda_{K_{s}}\right)^{-1.53}-1\right]} \\
& =\frac{(1.55-0.21)}{\left[(1.662 / 2.159)^{-1.53}-1\right]=2.73,}
\end{aligned}
$$

and from $E_{J-K}$

$$
\begin{aligned}
A_{K_{s}} & =\frac{E_{J-K}}{\left[\left(\lambda_{J} / \lambda_{K_{S}}\right)^{-1.53}-1\right]} \\
& =\frac{(4.62-0.89)}{\left[(1.2352 . / 2.159)^{-1.53}-1\right]=2.76 .}
\end{aligned}
$$

We adopt the average of these two inferred extinctions, $A_{K}=$ 2.74 , throughout the rest of this paper.

\subsection{Distance}

$\mathrm{OH} 25.25-0.16$ is an $\mathrm{OH}$ maser source centered about $5^{\prime \prime}$ north of the cluster center, at R.A. $=18^{\mathrm{h}} 37^{\mathrm{m}} 58^{\mathrm{s}} 27$, decl. $=$ $-06^{\circ} 52^{\prime} 28^{\prime \prime} 8$ (J2000.0). Blommaert et al. (1994) quoted a "typical" positional uncertainty for their sample of 4 "; it is unclear if this uncertainty applies to the position of $\mathrm{OH} 25.25-0.16$. The maser is roughly equidistant, $d \sim 9^{\prime \prime}$, from stars 9,13 , and 15 (see Fig. 2). These types of masers are often produced in the stellar winds of latest (M4-M5) RSGs (Lewis 1991), i.e., S Per, VX Sgr, NML Cyg, and VY CMa; see Humphreys (1975) for a review. We believe that the maser is likely produced by either star 9 or 13 , as 15 is too warm to be associated with such a maser. $\mathrm{OH}$ masers and RSGs are relatively rare, and we estimate a random probability of $10^{-10}$ of a chance alignment having the observed separation. Unlike most maser sources, this has only one velocity peak, at $102.2 \mathrm{~km} \mathrm{~s}^{-1}$. If we take this as the systemic velocity for the maser, and associate the source with the cluster, then we can estimate a distance to the cluster, assuming the Galactic rotation curve in Brand \& Blitz (1993). Doing so yields a distance of $5.8 \mathrm{kpc}$ (Fig. 10). This "near-side" distance places the RSG stars exactly at the luminosities expected from the isochrones (Fig. 6). If the stars were at the far-side solution implied by a fit to the Galactic rotation curve, they would then have $L \sim 10^{6} L_{\odot}$, a factor of 4 higher than for typical RSGs.

\subsection{Luminosities}

We can estimate the total luminosity of each star by integrating its spectral energy distribution. Being relatively cool, RSGs emit most of their radiation near $1 \mu \mathrm{m}$, although mid-infrared flux may contribute significantly in the case of photon reprocessing by circumstellar dust. Most of the RSG stars can be identified in observations from the Spitzer GLIMPSE survey (see Fig. 11), although several cannot because such a crowded field poses problems for the photometry extraction process. Figure 12 shows plots of the spectral energy distributions for the RSGs. As expected for cool stars, the magnitudes trend higher (fainter) at the shortest wavelengths.

Figure 13 shows blackbody fits to the dereddened fluxes from the 2MASS and Spitzer data. Each blackbody has been assigned 


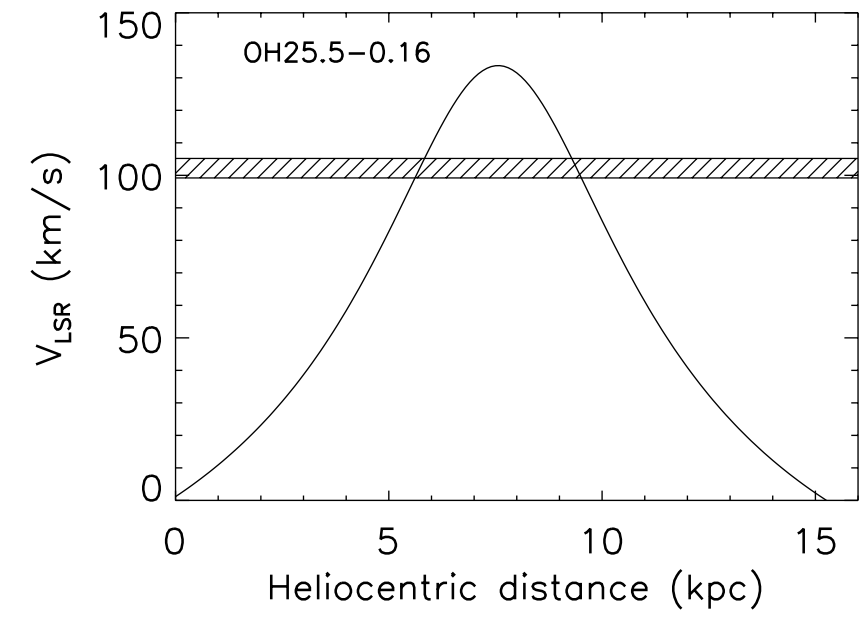

FIG. 10.-Plot of $V_{\mathrm{LSR}}$ vs. distance along the line of sight to the cluster, assuming the Galactic rotation curve in Brand \& Blitz (1993). The crosshatched region represents the velocity of the $\mathrm{OH} 25.5-0.16$ maser associated with the envelope of one of the RSGs in the cluster (star No. 9). The distance to the cluster is taken to be $5.8 \mathrm{kpc}$, corresponding to the "near-side" solution. Note that the "far-side" solution would require anomalously high luminosities for the RSGs.

the appropriate temperature corresponding to the spectral type (Levesque et al. 2005), although there may be evidence of a long-wavelength excess in some cases, i.e., 9,11 , and 12 . We computed the integrated luminosity under the blackbody curves, and the log of these values, in solar units, are plotted in the legends of the figure panels. All the luminosities are consistent with what would be expected for supergiants. Taken at face value, they suggest $M_{\mathrm{bol}} \sim-8$ to -9 , with correspondingly high implied initial masses of $M_{\text {initial }}=15-25 M_{\odot}$.

\section{DISCUSSION}

The cluster is extraordinary for its membership of 14 RSGs, more than any other cluster in the Galaxy. We show below that this implies a cluster mass of at least $20,000 M_{\odot}$, comparable to the masses of the most massive young clusters in the Galaxy. The implied age leads us to believe that the cluster is ripe for super-

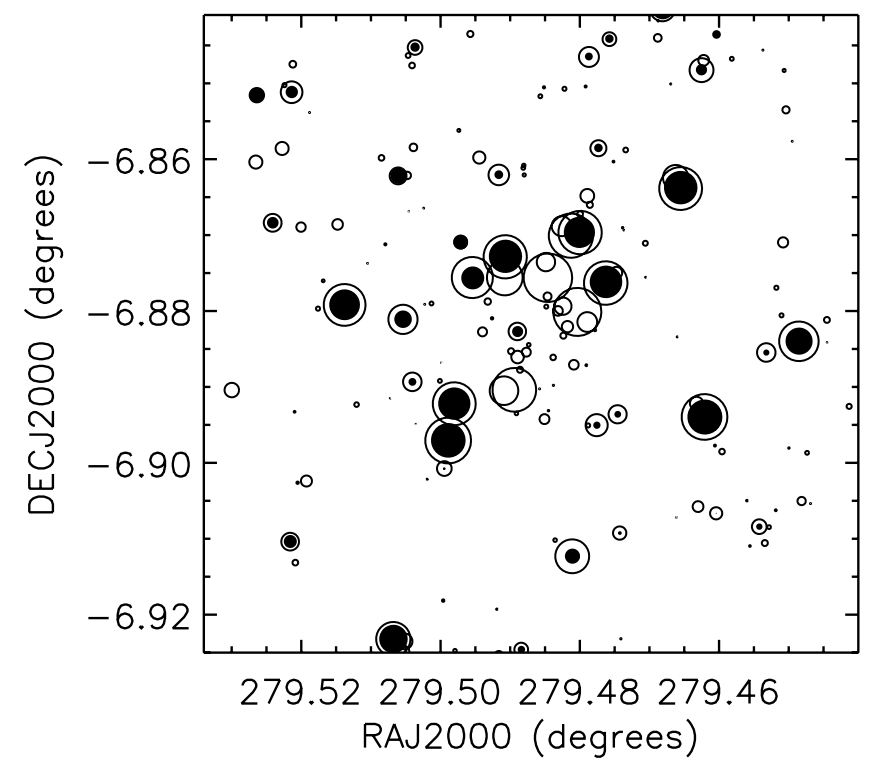

FIG. 11.-Supergiant cluster stars from 2MASS (open circles) and Spitzer GLIMPSE at $3.6 \mu \mathrm{m}$ ( filled circles). Symbol sizes scale with measured flux.

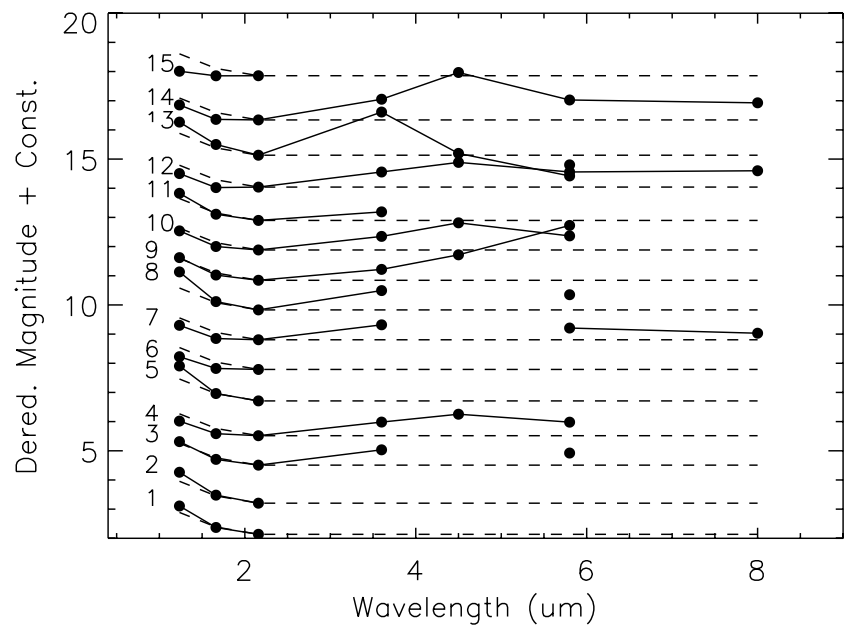

FIG. 12.-Dereddened magnitudes for cluster stars (circles with solid lines). The values have been shifted by their ID number for presentation purposes. The intrinsic magnitudes of RSG stars are overplotted (dashed lines). The upward trend of the magnitudes toward shorter wavelengths is expected for such cool stars on the magnitude system. Note that the differences between the observations and model are closely related to the inferred spectral types. For instance, the RSG model significantly underpredicts the flux at short wavelengths for star 15, a G6 I star, as would be expected.

nova activity. Indeed, we claim that the present-day supernova rate is consistent with the presence of high-energy objects in the field of the cluster.

Relatively little is known about this cluster. It is designated as candidate cluster 122 in the list of Dutra et al. (2003a), who noted that it is in/near the G25.253-0.150 and W42 regions, that it appears to be an infrared cluster, and that it is a loose collection of stars spanning approximately $4^{\prime}$ on the sky. The cluster is about $7^{\prime}$ to the southwest of the massive stars identified by Blum et al. (2000) in W42 (see Fig. 3). Our distance estimate places the RSG cluster within the rather large range of estimates for the distance of W42: 2.2, 3.7, 6.0, and $9.3 \mathrm{kpc}$ (Lester et al. 1985; Churchwell et al. 1990; Blum et al. 2000; Crowther \& Conti 2003). The bulk of the information known about this cluster is determined in this paper and described in the following sections.

The remainder of this section of the paper is devoted to establishing the characteristics of the RSG cluster in the context of massive young stellar clusters in the Galaxy. We find that the cluster is extraordinary for its content of RSGs and readily interpreted as a very massive cluster at an age ( $\sim 10 \mathrm{Myr})$ when it produces an extraordinary number of RSGs.

\subsection{Cluster Mass and Age}

The cluster mass can be estimated by fitting a reasonable IMF through the number of stars in the cluster with known initial masses, in this case, the RSGs. We can relate the RSG luminosities to initial masses by using stellar evolution models, age estimates, and assumptions about the metallicities and stellar mass-loss rates. To this end, we use a Monte Carlo simulation code that randomly draws initial stellar masses from a uniform distribution constrained by a Salpeter IMF (Salpeter 1955) and truncated at 0.8 and $150 M_{\odot}$ (Figer 2005). We then choose an isochrone to convert initial mass to present-day mass, temperature, luminosity, and absolute magnitude. For the figures in this paper, we choose the Geneva models with solar metallicity and canonical mass-loss rates. We convert to apparent magnitudes by adding the observed extinction and the distance modulus. Finally, we identify all the supergiant stars $\left(L / L_{\odot}>10^{4.5}\right)$ as falling into one of three categories: $\operatorname{RSG}(T<4500 \mathrm{~K})$, 

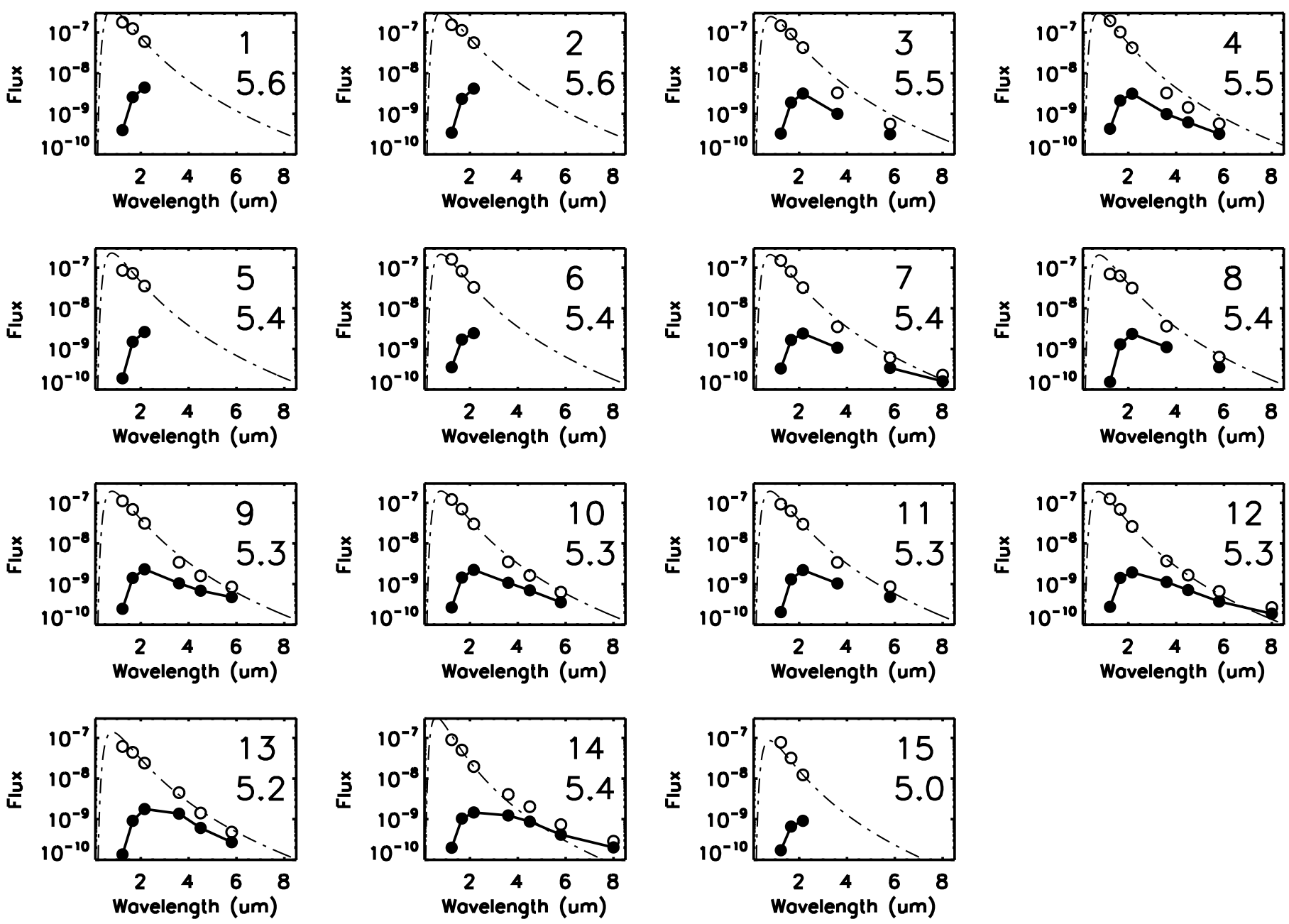

FIG. 13. - Photometry from 2MASS and Spitzer GLIMPSE surveys for the 14 supergiants in this paper. The solid lines are drawn through the observed fluxes ( filled circles), in units of ergs s $\mathrm{cm}^{-1} \mu \mathrm{m}^{-1}$. The dashed lines are drawn for blackbodies fitting the dereddened photometry (open circles) and having temperatures appropriate for the spectral types of the stars. The logs of the integrated luminosities, in solar units, under the blackbody curve are given in the legend.

yellow supergiant (YSG; $4500 \mathrm{~K}<T<10,000 \mathrm{~K}$ ), or BSG $(T>10,000 \mathrm{~K})$.

One set of sample clusters is shown in Figure 14 for ages of 10 and 14 Myr. As expected, we see that the ratio of BSGs to RSGs is lower for the older cluster. We are motivated to examine the behavior of the BSG to RSG ratio because it provides a critical test for stellar evolution models, and it determines the spectral appearance of integrated light from starburst populations (Langer \& Maeder 1995; Kippenhahn \& Weigert 1990; Maeder $\&$ Meynet 2000, 2001). The ratio for each trial is shown in Figure 15 for ages of 10 and 14 Myr. These plots demonstrate the statistical scatter induced by randomly sampling the IMF; a histogram of these values is shown in Figure 16. We can analyze the Monte Carlo results by examining statistical properties of the whole sample. For instance, Figure 17 shows the number of RSGs as a function of age for a cluster with initial mass of 30,000 $M_{\odot}$, and the associated ratio of BSGs to RSGs, both computed as the median over the set of sample clusters. We see that the number of RSGs peaks at $\approx 12 \mathrm{Myr}$, and the ratio of BSGs to RSGs is steadily decreasing as a function of age. The implied initial cluster mass is shown in Figure 18, assuming a cluster having 14 RSGs.

The cluster mass and stellar luminosities depend on age. Figure 19 shows a plot of $M_{K}$ versus cluster age for the most luminous and least luminous RSGs in a cluster demonstrating this behavior. From inspection of this figure, it appears that the high luminosities for the stars in the RSG cluster are consistent with a relatively young age $(\sim 7-12 \mathrm{Myr})$.

Several things are immediately apparent from these models. First, they require that the initial cluster be quite massive, at least $20,000 M_{\odot}$, and perhaps as high as $100,000 M_{\odot}$, where the actual value primarily depends on age. Second, there is a large brightness gap between the RSGs and the next brightest group of stars in the CMD, matching the observations. Finally, the BSG/RSG ratio can vary just from statistical sampling effects. For instance, it would be quite plausible to observe a cluster with $\approx \pm 20 \%$ of the median ratio, even if the stellar evolution models are perfectly accurate for that cluster. Of course, this effect scales strongly with the number of stars in the cluster; i.e., the standard deviation of the histogram scales roughly inverse linearly with the total initial cluster mass.

We also examine trends with metallicity. We find that twice solar metallicity model clusters produce more RSGs; i.e., the initial cluster masses might be only $\sim 20,000 M_{\odot}$ for all ages, whereas the BSG to RSG ratio is roughly half of that for solar metallicity.

Our large cluster mass estimate depends critically on the large number of RSGs in the cluster. There are two possibilities that could reduce the estimated total mass of the cluster: binarity/ multiplicity and noncoevality. Some objects could be multiple (although even then, the large gap in $K_{s}$ between the RSGs and 

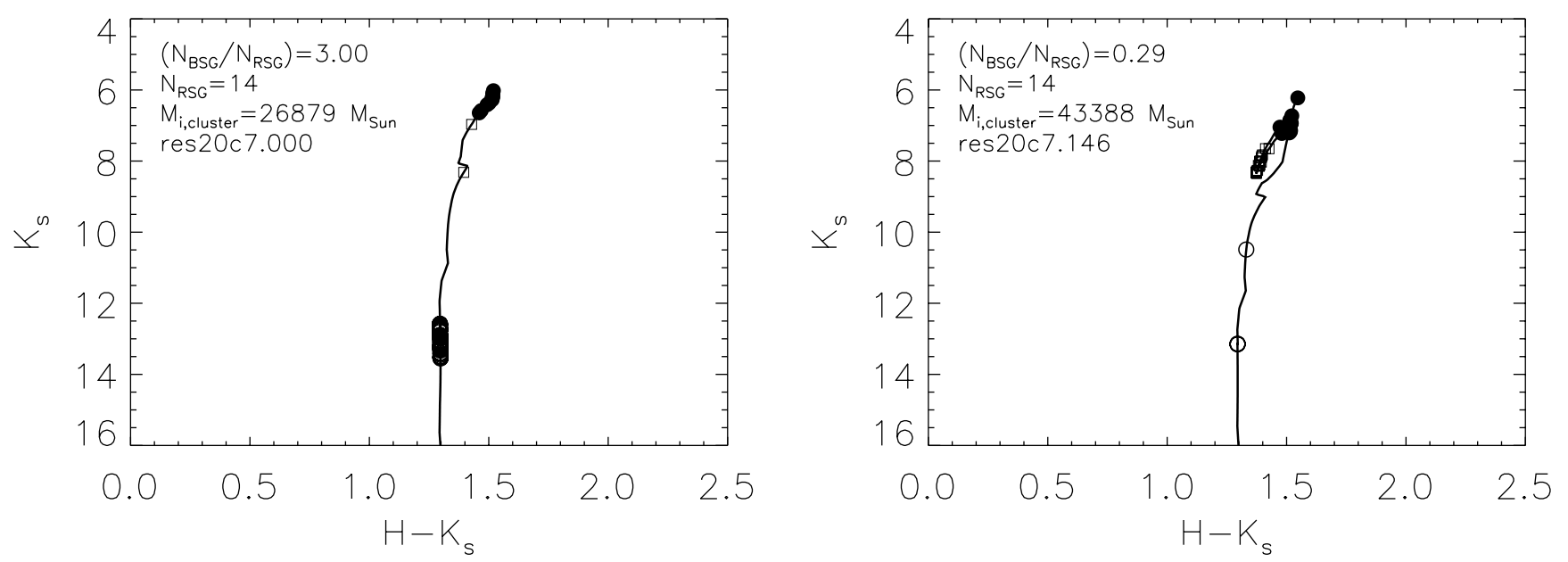

FIg. 14.- Sample CMDs from a Monte Carlo simulation for ages of $10 \mathrm{Myr}$ (left) and $14 \mathrm{Myr}$ (right), assuming the nonrotating Geneva models with solar metallicity and the canonical mass-loss rates. The symbols at the upper portion of the diagram are RSGs ( filled circles), and those near the bottom are BSGs (open circles). YSGs are designated by squares.
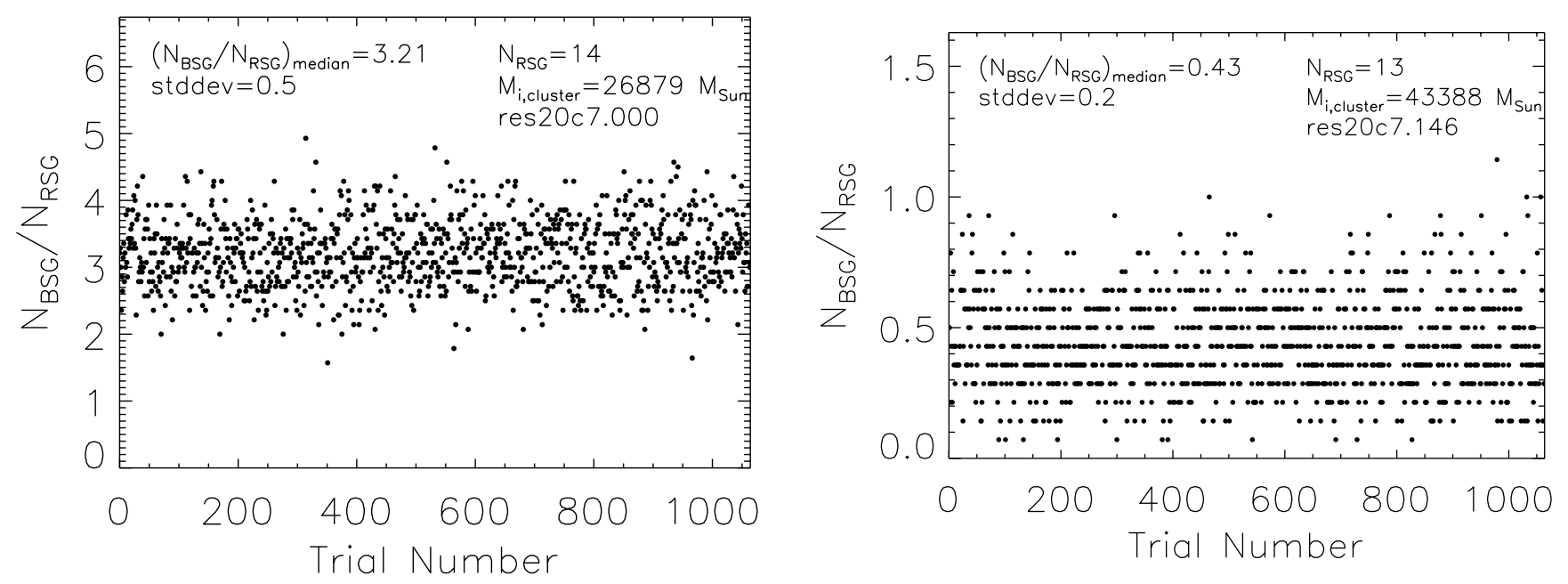

FIG. 15.- Ratio of BSGs to RSGs in sample trials from the Monte Carlo simulation. The cluster masses are randomly drawn from a distribution constrained to follow a Salpeter IMF such that the median number of RSGs is equal to 14, the observed value in the cluster. The plots are for ages of $10 \mathrm{Myr}$ (left) and $14 \mathrm{Myr}$ (right). The legend gives the median value of the BSG to RSG ratio, the median number of RSGs, the total estimated initial cluster mass, and the model isochrone file name.


FIG. 16.- Histogram of the sample defined in Fig. 15. The plots are for ages of $10 \mathrm{Myr}($ left $)$ and $14 \mathrm{Myr}$ (right). 

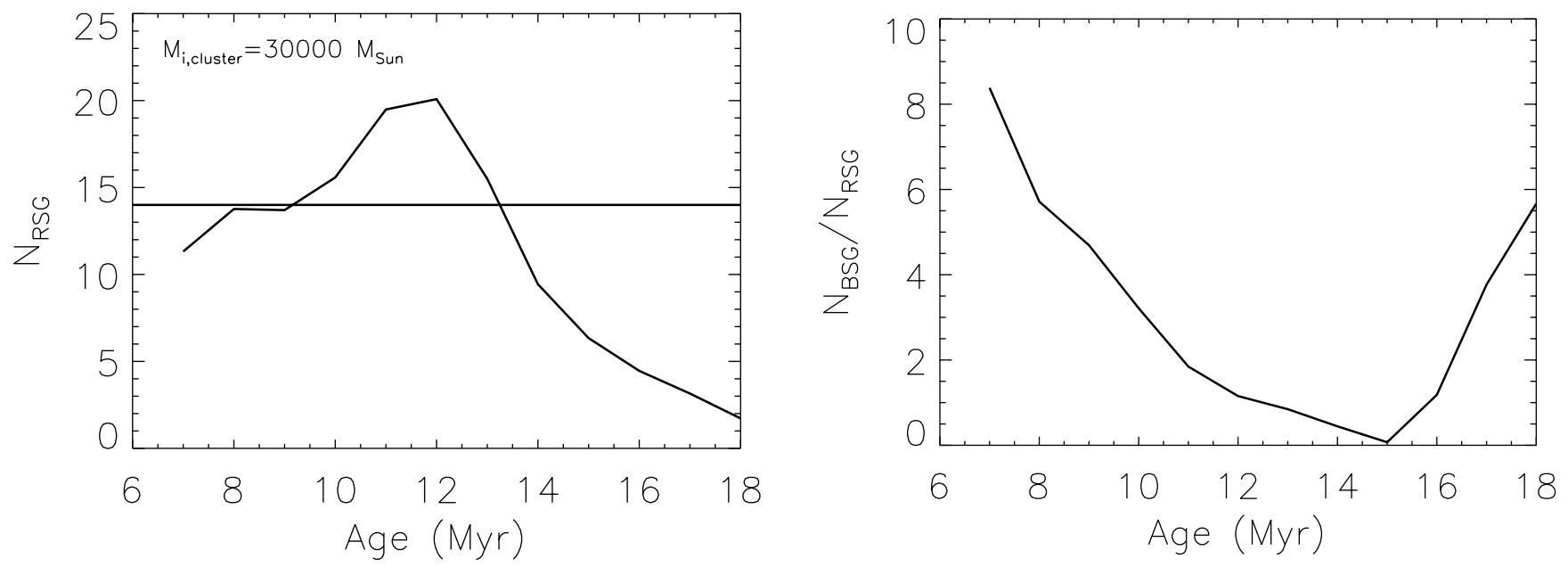

FIG. 17. - Progression of the number of RSGs (left $)$ and the ratio of BSG to RSGs (right) as functions of age for an initial cluster mass of $30,000 M_{\odot}$, assuming the nonrotating Geneva models with solar metallicity and the canonical mass-loss rates. The plot of RSGs can be scaled vertically as a linear function of initial cluster mass to match the number of observed RSGs indicated by the horizontal line.

the main sequence indicates that most of the bright sources would be RSGs, but not as extreme). Without higher resolution spectroscopy, it is difficult to asses the impact of this possibility. It is also possible that the bright stars were formed in several bursts, or via continuous star formation, over a substantial timescale. Figure 19 implies that the RSG progenitors could come from initial stellar masses spanning a range of $25-18.5 M_{\odot}$ for an age range spanning $2 \mathrm{Myr}$. While this age spread is typical for errors in estimating a cluster age, it would imply an extended star formation episode.

\subsection{High-Energy Objects near the RSG Cluster}

The field surrounding the cluster contains a number of highenergy sources, and Figures 2 and 3 show their spatial relationships. In this section, we interpret these sources as potential indicators of recent supernova activity in the cluster.

The Advanced Satellite for Cosmology and Astrophysics (ASCA) mission identified several sources in the field (Bamba et al. 2003). The center of the error circle for AX J1838.0-0655 is located to the south of the cluster center, and its flux in the $0.7-10 \mathrm{keV}$ energy band is $1.1 \times 10^{-11}$ ergs $\mathrm{cm}^{-2} \mathrm{~s}^{-1}$. The visual extinction to the source is estimated to be $A_{V}=36.3$ (Malizia et al. 2005), somewhat higher than the value we find for the RSG cluster



FIG. 18.- Implied initial cluster mass as a function of time, assuming a cluster having 14 RSGs and the Geneva models with solar metallicity.
$\left(A_{V}=2.74 / 0.112=24.5\right)$. Another X-ray source, AX J1837.50653 , is located to the west by $5^{\prime}$ (Bamba et al. 2003).

The International Gamma-Ray Astrophysics Laboratory (INTEGRAL) observatory also observed a source in the field (Malizia et al. 2005), to the south and east of the cluster center. Its flux in the $20-300 \mathrm{keV}$ energy range is $9 \times 10^{-11} \mathrm{ergs} \mathrm{cm}^{-2}$ $\mathrm{s}^{-1}$. Given the relatively large error circle for this source, it is possible that the source of the flux is located somewhere in the cluster.

The High Energy Spectroscopic System (HESS) telescopes detected high-energy $\gamma$-rays $(E>100 \mathrm{GeV})$ from HESS J1837069, which is located to the southwest of the cluster (see Fig. 3), at R.A. $=18^{\mathrm{h}} 37^{\mathrm{m}} 42^{\mathrm{s}} .7$ and decl. $=-6^{\circ} 55^{\prime} 39^{\prime \prime} 0$ (J2000.0), with a size of $7^{\prime}$ and a positional uncertainty of $1^{\prime}-2^{\prime}$ (Aharonian et al. 2005). We estimate a random chance alignment of the cluster and source of $\approx 0.05 \%$. The HESS source has a flux of $9 \times 10^{-12} \mathrm{ergs}$ $\mathrm{cm}^{-2} \mathrm{~s}^{-1}$ above $200 \mathrm{GeV}$, or $13.4 \%$ of that from the Crab Nebula. Assuming that the HESS source is associated with the

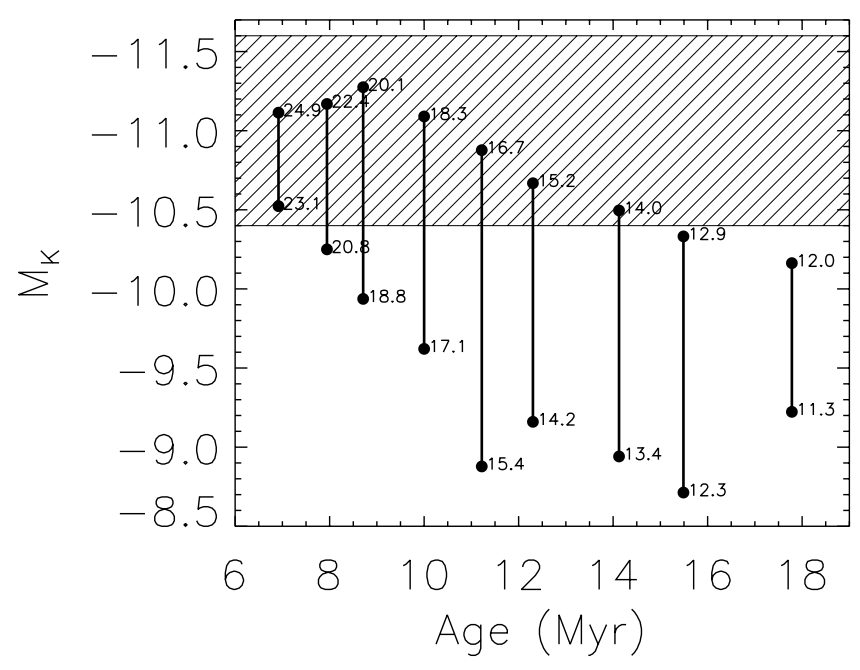

FIG. 19.-Plot of $M_{K}$ vs. cluster age for the most luminous and least luminous RSGs in a cluster, according to the Geneva models with solar metallicity and the canonical mass-loss rates. Initial stellar masses are plotted as data labels. Stars in the RSG cluster have $M_{K} \sim-10.5$ to -11.5 , as shown in the crosshatched region. Variations in metallicity or mass-loss rate do not significantly alter the trend that the observations are best fit by a relatively young cluster. 
RSG cluster, it has a luminosity of $(5800 / 1930) \times 13.4 \%=120 \%$ of that of the Crab. This would suggest that a recent supernovae in the cluster is responsible for this source. Aharonian et al. (2005) noted that the HESS object is likely a supernovae remnant or pulsar wind-driven nebula.

We believe that the high-energy sources are physically related to the RSG cluster. Indeed, there is precedence for associating extended high-energy sources with a cluster of massive stars, i.e., in CYG OB2 (Aharonian et al. 2002). Malizia et al. (2005) noted that AX J1838.0-0655 is very near to a bright mid-infrared source detected by MSX (MSX6C G025.2454-00.1885) but that the latter is likely too bright to be consistent with the large reddening inferred for the former. We argue the opposite. The $M S X$ source is the cluster of RSGs, and it is as bright as expected for such an object that is heavily extincted. Therefore, we associate the high-energy objects with the cluster. If we associate the ASCA source (AX J1838.0-0655), the INTEGRAL source, and the HESS source, with the same object, then the total X-ray, $\gamma$-ray, and TeV luminosities are 4.4, 36, and $3.6 \times 10^{34} \mathrm{ergs} \mathrm{s}^{-1}$ (Malizia et al. 2005), assuming $d=5800$ pc.

There are several other high-energy sources near the cluster that are either not related, or whose association with the cluster is uncertain. PSR J1837-06 is located $11^{\prime}$ to the west of the cluster, at R.A. $=18^{\mathrm{h}} 37^{\mathrm{m}} 14^{\mathrm{s}} .65$, decl. $=-6^{\circ} 53^{\prime} 2^{\prime \prime}$. 1 (J2000.0). It has a period of $1.9 \mathrm{~s}$ and a distance of $\sim 5 \mathrm{kpc}$, a spin-down age $\tau=$ $39 \mathrm{Myr}$, where the spin-down age is defined to be $P / 2 \dot{P}$, and a spin-down flux of $1.5 \times 10^{-15} \mathrm{ergs} \mathrm{cm}^{-2} \mathrm{~s}^{-1}$ (Malizia et al. 2005) or $2.5 \times 10^{26} \mathrm{ergs} \mathrm{s}^{-1}$ at 5800 pc. The spin-down rate is $\dot{P}=7.7 \times$ $10^{-16} \mathrm{~s} \mathrm{~s}^{-1}$. The magnetic field strength is $3.2 \times 10^{19}(P \dot{P})^{0.5}$, or $1.2 \times 10^{12} \mathrm{G}$ (not enough to be a magnetar, $\sim 10^{14} \mathrm{G}$ ). Note that this PSR is likely not associated with the HESS source (Malizia et al. 2005), and it is quite a distance from the cluster. The hard $\mathrm{X}$-ray source, G25.5+0.0, is nearby and thought to be a supernova remnant (SNR; Bamba et al. 2003). Given its projected distance from the cluster, it is unclear whether it is related.

\subsection{Radio/IR Objects near the RSG Cluster}

There are a number of radio objects in the field, as shown in Figure 2. Altenhoff et al. (1979) identified G025.252-0.150 as a bright $6.1 \mathrm{~cm}$ source $(0.81 \mathrm{Jy})$ centered on the cluster field. Helfand et al. (1989a) resolved this source, at 20 and $90 \mathrm{~cm}$, into three nonthermal sources and regarded them as likely active galactic nuclei, although they noted the nearby "unrelated" IRAS source just $1^{\prime}$ to the north (IRAS 18352-0655); the IRAS source represents the cumulative flux from the RSGs. Figure 20 shows flux from the radio objects in the field at $20 \mathrm{~cm}$ from the Galactic Plane Survey (White et al. 2005; Helfand et al. 2006). ${ }^{9} \mathrm{We}$ discuss these three sources in detail below. In general, the sources are nonthermal in nature, supporting our claim that they are related to supernovae activity from the cluster. They are also spatially coincident with the cluster, and similar radio sources are not seen within a one square degree field in the MAGPIS data, again supporting a connection between the radio sources and the cluster.

Zoonematkermani et al. (1990) identified GPSR 25.2670.161 , near star 6 , in $21.4 \mathrm{~cm}$ observations using the Very Large Array in the B configuration, i.e., positional accuracy of $\sim 3^{\prime \prime}$. This is the same object as GPSR5 25.266-0.161, identified by Becker et al. (1994) at $6 \mathrm{~cm}$. The source has a spectral index of $\alpha=\log (254 \mathrm{mJy} / 551 \mathrm{mJy}) / \log (5 \mathrm{GHz} / 1.4 \mathrm{GHz})=-0.60$ (Helfand \& Chanan 1989b; Becker et al. 1994).

\footnotetext{
${ }^{9}$ The data for this figure are from MAGPIS; see http://third.ucllnl.org/gps/ index.html.
}

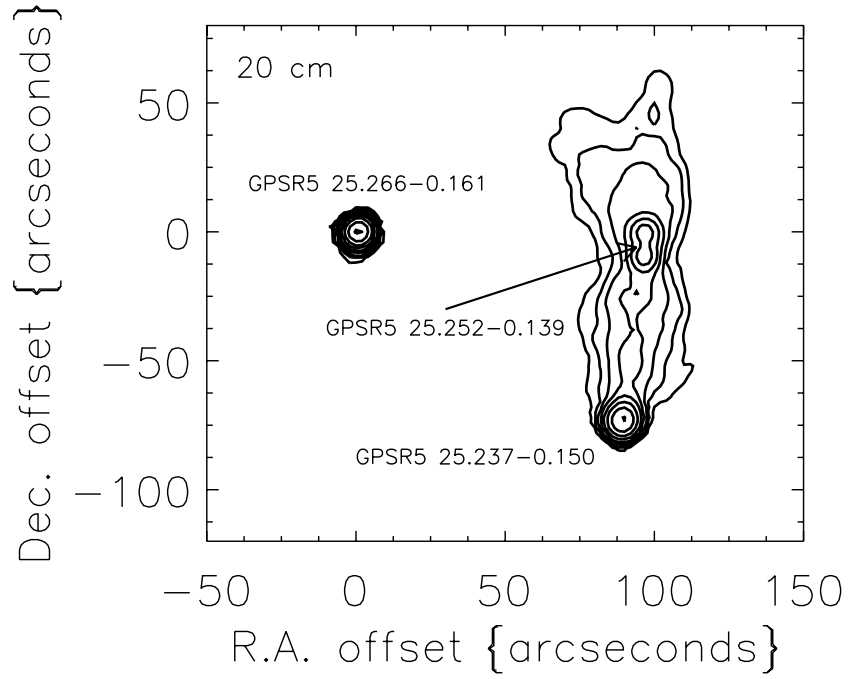

FIG. 20.-Contour plot of radio flux at $20 \mathrm{~cm}$ (White et al. 2005; Helfand et al. 2006) within the cluster field (see Fig. 2). The contours are drawn at flux levels, in millijanskys per beam: $2,4,8,16,32,64,128,256,512$, and 1024 . The beam size is $6.2 \times 5$ ". 4 , with the long axis along the north-south direction.

Becker et al. (1994) associated two separate sources with GPSR5 25.252-0.139, having spectral indices of 0.14 and $<-1.6$, and separated by $7^{\prime \prime}$ in the north-south direction. These sources appear to be at the center of emission that extends $1^{\prime}$ to the north and south at $20 \mathrm{~cm}$. It is intriguing to note that its size, $\sim 1^{\prime} \times 2^{\prime}$, is roughly the same the Crab Nebula would have at $5800 \mathrm{pc}$. The size of the $\mathrm{Crab}$ is $\sim 4^{\prime} \times 6^{\prime}$, and it is at a distance of 1930 pc. At 5800 pc, its apparent size would be $\sim 1 ! 3 \times 2$ ' $^{\prime} 0$. The extended emission does not appear at $6 \mathrm{~cm}$ in the Galactic Plane Survey at a level above $1 \mathrm{mJy}^{\text {beam }}{ }^{-1}$, or $28 \mu \mathrm{Jy} \operatorname{arcsec}^{-2}$. This nondetection suggests a spectral index of $\alpha<\log (0.028 \mathrm{mJy} /$ $149 \mathrm{mJy}) / \log (5 \mathrm{GHz} / 1.4 \mathrm{GHz})=-1.32$; i.e., the source of the extended emission is nonthermal. We suggest that this source may be a supernovae remnant, similar to the Crab.

Becker et al. (1994) also identified GPSR5 25.237-0.150 about $1^{\prime}$ to the south of star 4 and GPSR 25.267-0.161. This source has a spectral index of $\log (111 \mathrm{mJy} / 238 \mathrm{mJy}) / \log (5 \mathrm{GHz} /$ $1.4 \mathrm{GHz})=-0.60$.

The Culgoora far-infrared/submillimeter circular array identified a prominent source in the field, Cul 1835-069 (Slee 1995), with an effective beam diameter of several arcminutes. The spectral index at these wavelengths is -0.70 . Given the similarity of this spectral index to that at centimeter wavelengths, we assume that the radio and submillimeter objects are, in fact, the same, and different from the flux from the RSGs ( $\alpha=2$ for the RayleighJeans tail of a blackbody). Volk \& Cohen (1989) identified NSV 11126 as having an unusual IRAS Low-Resolution Spectrometer spectrum at mid-infrared wavelengths. This source likely represents light from the RSGs, as do IRAS 18352-0655 and RAFGL 5268S. As already discussed, the cluster is identified in MSX images at mid-infrared wavelengths (see Fig. 3).

\subsection{End States and Initial Stellar Mass}

A number of studies predict that the most massive stars will largely evaporate in their lifetimes, leaving relatively low mass objects before they explode as supernovae. For example, Heger et al. (2003) predicted that stars with solar metallicities and $M_{\text {initial }}>50 M_{\odot}$ will collapse as neutron stars, whereas those with $25 M_{\odot}>M_{\text {initial }}>50 M_{\odot}$ collapse as black holes. Lower mass stars, $9 M_{\odot}>M_{\text {initial }}>25 M_{\odot}$, will collapse as neutron 




FIG. 21.-Mean time between supernovae for a cluster with 14 RSGs vs. age.

stars. These models are difficult to test. A successful test requires that an object have a well-determined initial mass and that the nature of the end state is well known. Of course, once the object has reached the end state, it is difficult to independently infer the initial mass of that object. In a few cases, a supernova progenitor has been observed, but even in these cases, there is usually a very poor correlation between progenitor and initial mass (Smartt et al. 2004; van Dyk 2005). These progenitors have always been either RSGs or BSGs, and it is difficult to uniquely determine initial masses for such stars.

One promising approach to relate end state to initial mass is to find a recent supernovae remnant and relate the object to the present-day upper-mass cutoff in the coeval cluster in which it was formed. This approach has been successfully used to relate magnetars to particularly massive progenitors $\left(M_{\text {initial }}>50 M_{\odot}\right.$; Figer et al. 2005; Muno et al. 2006), and an alternate method confirms these results (Gaensler et al. 2005). In this regard, the RSG cluster might be an ideal test bed for such a connection. Given that the most massive stars are RSGs, we expect there to be a very narrow range of masses defining the most massive stars in the cluster, although the precise value of that range will scale with age (Fig. 19). In any case, it is certainly likely to be somewhere between 12 and $25 M_{\odot}$, with a bias for a younger age and masses at the high end of the range.

We plan to obtain deeper observations in order to provide a tighter constraint on the masses of the stars (and, in turn, the cluster age). We also plan to determine the nature of the most likely compact object that might have been produced in the cluster.

We can estimate the supernova rate for this cluster as a function of assumed age. As with our previous simulations, we assume 14 RSGs and the Geneva models with solar metallicity and the canonical mass-loss rates. Figure 21 shows the average time between supernovae for clusters that have enough mass (Fig. 18) to produce $14 \mathrm{RSGs}$ at a given age. We find that there should be a supernova in the cluster every $\sim 40,000-80,000 \mathrm{yr}$, on average. This timescale is consistent with the presence of putative postsupernovae phenomena, i.e., X-ray and $\gamma$-ray sources, as found near the cluster.

\subsection{Comparison to Other Clusters with RSGs}

The cluster mass is extraordinary, perhaps trailing only Westerlund 1, as the most massive young cluster in the Galaxy (Clark et al. 2005). One difficulty in making cluster mass com-

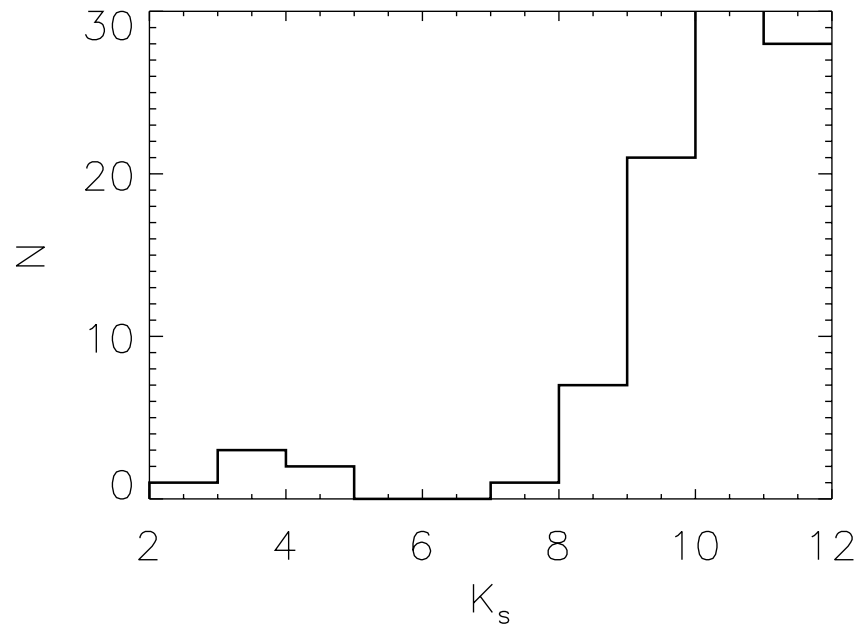

FIG. 22.-2MASS luminosity function of stars in the field containing NGC 7419. The sample has been culled of stars with reported errors greater than $0.1 \mathrm{mag}$ in $K_{s}$.

parisons is that all of the mass estimates are made by extrapolating an IMF down to masses below the observational limit. Only in the Arches cluster, near the Galactic center, do we directly count the majority of the stars in the cluster. In that case, the slope of the IMF, for stars currently in the cluster, deviates significantly from the Salpeter value (Figer et al. 1999a; Stolte et al. 2002). Thus, the initial cluster mass for the Arches cluster is inferred to be $\sim 10,000 M_{\odot}$, even though it currently has an estimated $160 \mathrm{O}$ stars (Figer et al. 2002). However, it is possible that the true initial mass was much higher and that the presentday cluster has been heavily evacuated of low-mass stars via $\mathrm{N}$-body interactions (Kim et al. 2000; Portegies Zwart et al. 2002). If we simply integrate a Salpeter IMF from the high-mass stars down to $0.5 M_{\odot}$, we find a mass of $50,000 M_{\odot}$. Using this technique, we would find similar values for the Quintuplet cluster and the cluster in the central parsec of the Galaxy (Figer et al. 1999b). For Westerlund 1, we would find a mass of $\sim 10^{5} M_{\odot}$ (Clark et al. 2005). Given the uncertainties of all the estimates involved, we claim that the cluster of RSGs is in a class of massive young stellar clusters containing the aforementioned clusters. Until these clusters are observed down to $\sim 1 M_{\odot}$, and the effects of dynamical evolution better understood, it will be difficult to know their true initial mass-rank ordering.

In any case, this group of clusters substantially overlaps with the low-mass end of the super star clusters often found in interacting galaxies (Whitmore et al. 1999) and the low-mass end of the Galactic globular cluster population (Mandushev et al. 1991). This indicates that the cluster formation mechanisms currently found in the Galaxy might be similar to those in interacting systems or in the young Galaxy, albeit at a much subdued level.

\subsection{Comparison to NGC 7419}

One can compare this cluster to NGC 7419 , previously thought to be the richest cluster of red supergiants in the Galaxy, with five. Caron et al. (2003) noted that the BSG to RSG ratio in this cluster is lower than that for any known cluster in the Galaxy, at least as low as 1:5, and may be due to the often mentioned effects of the observed rapid stellar rotation in the stars (Maeder $\&$ Meynet 2001). Model isochrones successfully match the observations for an age of $15 \mathrm{Myr}$. This cluster has an estimated metallicity near the solar value. 



FIG. 23.-2MASS CMD of stars in the field containing NGC 7419. The RSGs are represented by filled circles, and the fainter field stars are designated by open circles. A $15 \mathrm{Myr}$ isochrone is overplotted, assuming the Geneva models with solar metallicity, a distance of $2.3 \mathrm{kpc}, A_{K_{s}}=0.7$, and the reddening law of Rieke et al. (1989). The sample has been culled of stars with reported errors greater than $0.1 \mathrm{mag}$ in $K_{s}$.

Figures 22, 23, and 24 show 2MASS data for NGC 7419 that are similar to those seen for the RSG cluster discussed in this paper. That is, there appears to be a large gap in $K_{s}$ between the RSGs and the bulk of the fainter stars in the cluster.

We obtained a spectrum of one of the RSG stars (Fig. 25), which indicates a spectral type of M0 I, compared to M2 I, given by Blanco et al. (1955). This validates our method to an accuracy within a few subtypes.

Assuming a distance of $2 \mathrm{kpc}$ to NGC 7419, the average of the values in Caron et al. (2003) and Beauchamp et al. (1994), and $A_{K}=0.67 \times 0.112$ (Beauchamp et al. 1994), we find $M_{K}=$ -7.6 for the four fainter RSGs and $M_{K}=-9.5$ for the brightest member. The extraordinary infrared brightness of the brightest member can be attributed to its very red spectral energy distribution (M7.5 I); note that this star also produces an $\mathrm{OH}$ maser, just as we find for one of the stars in our cluster. In comparison, the stars in our cluster have $M_{K} \sim-10.4$ to -11.6 (see Table 1). Beauchamp et al. (1994) noted that the RSGs in NGC 7419 are faint compared to those found elsewhere in the Galaxy that typ-

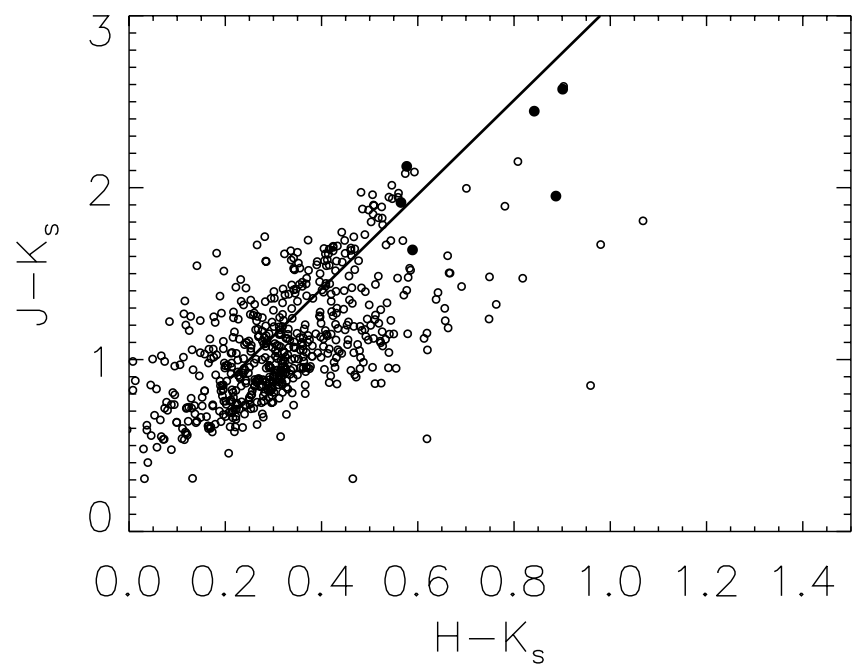

ically have $M_{K} \sim-10.5$. We interpret the data to indicate that our cluster has relatively massive stars and is thus younger than $15 \mathrm{Myr}$, the age of NGC 7419 (Caron et al. 2003). This is consistent with Figure 19, which shows that $M_{K}$ progresses from $\sim-11.2$ to -10.2 for the brightest RSG in a cluster with an $\tau_{\text {age }} \sim 8-18$ Myr, respectively.

\subsection{Comparison to $h$ and $\chi$ Persei and the Per OB1 Association}

The double cluster $\mathrm{h}$ and $\chi$ Per, and the surrounding Per OB1 association, collectively contain $\sim 20$ RSGs. While containing more RSGs than in the cluster we describe, the Perseus RSGs were likely formed in several episodes spanning on the order of $10 \mathrm{Myr}$ (Slesnick et al. 2002). Because of this, it is difficult to directly analyze the properties of the full Perseus RSG population without directly knowing which members belong to the various star formation episodes. Individually, $\mathrm{h}$ and $\chi$ Per are each $\sim 10 \mathrm{Myr}$ old and contain $\sim 5000 M_{\odot}$ in stellar mass (Bragg \& Kenyon 2005), considerably less than estimated for our cluster.



FIG. 24.-2MASS color-color diagrams of stars in the field containing NGC 7419. The RSGs are represented by filled circles, and the fainter field stars are designated by open circles. A reddening vector is plotted from the expected intrinsic colors of RSGs, assuming the extinction law of Rieke et al. (1989). The sample has been culled of stars with reported errors greater than $0.1 \mathrm{mag}$ in $K_{s}$. 




FIG. 25.-IRMOS spectrum of NGC 7419d. Blanco et al. (1955) gave a spectral type of M2 I using optical data, whereas we estimate M0 I with our method.

We presume this is consistent with the relatively low number of bona fide RSGs (only one) that can directly be attributed to either of the two double clusters, whereas our cluster has 14.

\section{CONCLUSIONS}

We have identified one of the most massive young stellar clusters in the Galaxy, containing 14 RSGs, more than in any cluster in the LMC, SMC, or the Galaxy. From an analysis of the estimated luminosities of the RSGs, we estimate an age of $\sim 10 \mathrm{Myr}$. We further note the rich collection of radio, X-ray, and $\gamma$-ray objects coincident with the location of the cluster that can be understood as a natural consequence of massive stars progressing to supernovae, as predicted by stellar evolution models. The RSGs in this cluster are likely direct progenitors of supernovae and provide a particularly ripe sample for studies relating com- pact objects to initial stellar masses. We note that one particular object, GPSR $525.252-0.139$, is a likely SNR, with properties similar to the Crab Nebula. In order to test stellar evolution models, we plan to obtain deeper observations of the RSG cluster to estimate the IMF slope, and BSG to RSG ratio. We also plan to obtain spectroscopic observations in order to determine if the rotation rates of the RSGs are consistent with theoretical expectations for such a rich cluster of these relatively rare stars.

Note added in manuscript.-Trejo \& Rodriguez (2006) convincingly show that the bright nonthermal radio sources discussed in this paper are extragalactic in nature.

We appreciate useful discussions with Mike Muno, Andy Fruchter, Brad Whitmore, Claus Leitherer, Nolan Walborn, Rick White, Roberta Humphreys, Phil Massey, Norbert Langer, Tony Moffat, and Andre Maeder. We thank the Spitzer GLIMPSE team, especially Ed Churchwell, Marilyn Meade, Brian Babler, Remy Indebetouw, and Barbara Whitney, for making images available before public release. We also thank the referee for useful comments, especially regarding the distance to W42. The material in this paper is based on work supported by NASA under award NNG 05-GC37G, through the Long Term Space Astrophysics program. F. N. acknowledges PNAYA 2003-02785-E and AYA 2004-08271-C02-02 grants and the Ramon y Cajal program. IRMOS is supported by NASA James Webb Space Telescope, NASA Goddard Space Flight Center, STScI DDRF, and KPNO. This research has made use of data obtained from the High Energy Astrophysics Science Archive Research Center (HEASARC), provided by the NASA/GSFC. This work made use of data from the Multi-Array Galactic Plane Imaging Survey (MAGPIS; White et al. 2005; Helfand et al. 2006), and the MAGPIS Web site, http:// third.ucllnl.org/gps/index.html.
Aharonian, F., et al. 2002, A\&A, 393, L37 2005, Science, 307, 1938

Altenhoff, W. J., Downes, D., Pauls, T., \& Schraml, J. 1979, A\&AS, 35, 23

Bamba, A., Ueno, M., Koyama, K., \& Yamauchi, S. 2003, ApJ, 589, 253

Beauchamp, A., Moffat, A. F. J., \& Drissen, L. 1994, ApJS, 93, 187

Becker, R. H., White, R. L., Helfand, D. J., \& Zoonematkermani, S. 1994, ApJS, 91, 347

Benjamin, R. A., et al. 2003, PASP, 115, 953

Bica, E., Bonatto, C., \& Dutra, C. M. 2004, A\&A, 422, 555

Bica, E., Dutra, C. M., \& Barbuy, B. 2003a, A\&A, 397, 177

Bica, E., Dutra, C. M., Soares, J., \& Barbuy, B. 2003b, A\&A, 404, 223

Blanco, V., Nassau, J. J., Stock, J., \& Wehlau, W. 1955, ApJ, 121, 637

Blommaert, J. A. D. L., van Langevelde, H. J., \& Michiels, W. F. P. 1994, A\&A, 287, 479

Blum, R. D., Conti, P. S., \& Damineli, A. 2000, AJ, 119, 1860

Borissova, J., Ivanov, V. D., Minniti, D., Geisler, D., \& Stephens, A. W. 2005, A\&A, 435, 95

Bragg, A. E., \& Kenyon, S. J. 2005, AJ, 130, 134

Brand, J., \& Blitz, L. 1993, A\&A, 275, 67

Caron, G., Moffat, A. F. J., St-Louis, N., Wade, G. A., \& Lester, J. B. 2003, AJ, 126,1415

Churchwell, E., Walmsley, C. M., \& Cesaroni, R. 1990, A\&AS, 83, 119

Clark, J. S., Negueruela, I., Crowther, P. A., \& Goodwin, S. P. 2005, A\&A, 434, 949

Crowther, P. A., \& Conti, P. S. 2003, MNRAS, 343, 143

Dutra, C. M., Bica, E., Soares, J., \& Barbuy, B. 2003a, A\&A, 400, 533

Dutra, C. M., Ortolani, S., Bica, E., Barbuy, B., Zoccali, M., \& Momany, Y. 2003b, A\&A, 408, 127

Elias, J. H., Frogel, J. A., \& Humphreys, R. M. 1985, ApJS, 57, 91

Figer, D. F. 2004, in ASP Conf. Ser. 322, The Formation and Evolution of Massive Young Star Clusters, ed. H. J. G. L. M. Lamers, L. J. Smith, \& A. Nota (San Francisco: ASP), 49

. 2005, Nature, 434, 192

\section{REFERENCES}

Figer, D. F., Kim, S. S., Morris, M., Serabyn, E., Rich, R. M., \& McLean, I. S. 1999a, ApJ, 525, 750

Figer, D. F., McLean, I. S., \& Morris, M. 1999b, ApJ, 514, 202

Figer, D. F., Najarro, F., Geballe, T. R., Blum, R. D., \& Kudritzki, R. P. 2005, ApJ, 622, L49

Figer, D. F., et al. 2002, ApJ, 581, 258

Gaensler, B. M., et al. 2005, Nature, 434, 1104

Garmany, C. D., \& Stencel, R. E. 1992, A\&AS, 94, 211

Heger, A., Fryer, C. L., Woosley, S. E., Langer, N., \& Hartmann, D. H. 2003, ApJ, 591, 288

Helfand, D. J., Becker, R. H., White, R. L., Fallon, A., \& Tuttle, S. 2006, AJ, 131,2525

Helfand, D. J., \& Chanan, G. A. 1989b, AJ, 98, 1652

Helfand, D. J., Velusamy, T., Becker, R. H., \& Lockman, F. J. 1989a, ApJ, 341, 151

Hirschi, R., Meynet, G., \& Maeder, A. 2004, A\&A, 425, 649

Humphreys, R. M. 1975, PASP, 87, 433

- 1978, ApJS, 38, 309

1979a, ApJ, 231, 384

1979b, ApJS, 39, 389

Humphreys, R. M., \& McElroy, D. B. 1984, ApJ, 284, 565

Humphreys, R. W. 1970, ApJ, 160, 1149

Ivanov, V. D., Borissova, J., Bresolin, F., \& Pessev, P. 2005, A\&A, 435, 107

Kharchenko, N. V., Piskunov, A. E., Röser, S., Schilbach, E., \& Scholz, R.-D. 2005, A\&A, 438, 1163

Kim, S. S., Figer, D. F., Lee, H. M., \& Morris, M. 2000, ApJ, 545, 301

Kippenhahn, R., \& Weigert, A. 1990, Stellar Structure and Evolution (Berlin: Springer)

Kleinmann, S. G., \& Hall, D. N. B. 1986, ApJS, 62, 501

Kroupa, P. 2002, Science, 295, 82

Langer, N., \& Maeder, A. 1995, A\&A, 295, 685

Leistra, A., Cotera, A. S., Liebert, J., \& Burton, M. 2005, AJ, 130, 1719

Lester, D. F., Dinerstein, H. L., Werner, M. W., Harvey, P. M., Evans, N. J., \& Brown, R. L. 1985, ApJ, 296, 565 
Levesque, E. M., Massey, P., Olsen, K. A. G., Plez, B., Josselin, E., Maeder, A., \& Meynet, G. 2005, ApJ, 628, 973

Lewis, B. M. 1991, AJ, 101, 254

MacKenty, J. W., et al. 2004, AAS Meeting, 205, 49.08

Maeder, A., \& Meynet, G. 2000, ARA\&A, 38, 143 2001, A\&A, 373, 555

Malizia, A., et al. 2005, ApJ, 630, L157

Mandushev, G., Staneva, A., \& Spasova, N. 1991, A\&A, 252, 94

Massey, P., \& Olsen, K. A. G. 2003, AJ, 126, 2867

McGregor, P. J., \& Hyland, A. R. 1984, ApJ, 277, 149

Muno, M. P., et al. 2006, ApJ, 636, L41

Portegies Zwart, S. F., Makino, J., McMillan, S. L. W., \& Hut, P. 2002, ApJ, 565,265

Price, S. D., Egan, M. P., Carey, S. J., Mizuno, D. R., \& Kuchar, T. A. 2001, AJ, 121, 2819

Rieke, G. H., Rieke, M. J., \& Paul, A. E. 1989, ApJ, 336, 752

Salpeter, E. E. 1955, ApJ, 121, 161

Schaerer, D., Charbonnel, C., Meynet, G., Maeder, A., \& Schaller, G. 1993, A\&AS, 102, 339
Skrutskie, M. F., et al. 1997, in The Impact of Large Scale Near-IR Sky Surveys, ed. F. Garzon et al. (Dordrecht: Kluwer), 25 2006, AJ, 131, 1163

Slee, O. B. 1995, Australian J. Phys., 48, 143

Slesnick, C. L., Hillenbrand, L. A., \& Massey, P. 2002, ApJ, 576, 880

Smartt, S. J., Maund, J. R., Hendry, M. A., Tout, C. A., Gilmore, G. F., Mattila, S., \& Benn, C. R. 2004, Science, 303, 499

Stolte, A., Grebel, E. K., Brandner, W., \& Figer, D. F. 2002, A\&A, 394, 459 Trejo, A., \& Rodriguez, L. F. 2006, Rev. Mex. AA, in press

van Dyk, S. D. 2005, in ASP Conf. Ser. 342, 1604-2004: Supernovae as Cosmological Lighthouses, ed. M. Turatto et al. (San Francisco: ASP), 115 Volk, K., \& Cohen, M. 1989, AJ, 98, 931

Walborn, N. R., Prevot, M. L., Prevot, L., Wamsteker, W., Gonzalez, R., Gilmozzi, R., \& Fitzpatrick, E. L. 1989, A\&A, 219, 229

White, R. L., Becker, R. H., \& Helfand, D. J. 2005, AJ, 130, 586

Whitmore, B. C., Zhang, Q., Leitherer, C., Fall, S. M., Schweizer, F., \& Miller, B. W. 1999, AJ, 118, 1551

Zoonematkermani, S., Helfand, D. J., Becker, R. H., White, R. L., \& Perley, R. A. 1990, ApJS, 74, 181 